\title{
Thermomagnetic properties of QCD
}

\author{
Christoph P. Hofmann \\ Facultad de Ciencias, Universidad de Colima, Bernal Díaz del Castillo 340, Colima C.P. 28045, Mexico
}

(Received 14 December 2020; accepted 28 June 2021; published 30 July 2021)

\begin{abstract}
We explore the low-energy regime of quantum chromodynamics subjected to an external magnetic field by deriving the two-loop representations for the entropy density and the finite-temperature magnetization within chiral perturbation theory (CHPT). At fixed temperature, the entropy density drops when the magnetic field becomes stronger. The magnetization induced at finite temperature is negative in the entire parameter region accessible by CHPT. We also point out that the enhancement of the finite-temperature part in the quark condensate is correlated with the decrease of the entropy density.
\end{abstract}

DOI: 10.1103/PhysRevD.104.014025

\section{INTRODUCTION}

The thermodynamic properties of quantum chromodynamics in a homogeneous external magnetic field have been explored by many authors. In the present study we focus on the entropy density and the finite-temperature magnetization ${ }^{1}$ - and furthermore shed light on the connection between the quark condensate and entropy density. Articles that also have discussed the dependence of entropy density and magnetization on temperature, magnetic field strength, and pion mass are: Refs. [1-5] based on lattice QCD, Refs. [6-9] based on the Nambu-Jona-Lasinio model and extensions thereof, as well as Refs. [10-23] that rely on yet other methods.

Still, in the regime of low temperatures and weak magnetic fields, a comprehensive investigation of entropy density and finite-temperature magnetization appears to be lacking. Here, within the framework of two-flavor chiral perturbation theory, we provide such a fully systematic analysis. Based on earlier work of the author, Refs. [2426], we derive the two-loop representations for the entropy density and the finite-temperature magnetization.

We find that the entropy density, at fixed temperature, decreases when the magnetic field becomes stronger and it also decreases when the masses of the pions grow. The impact of the magnetic field is most pronounced in the chiral limit. In the real world with pion masses fixed at

\footnotetext{
${ }^{1}$ By "finite-temperature magnetization" or "magnetization induced at finite temperature" we refer to the quantity $\mathfrak{M}_{T}$ that corresponds to the total magnetization with the zero-temperature portion $\mathfrak{M}_{0}$ subtracted: $\mathfrak{M}_{T}=\mathfrak{M}_{\text {tot }}-\mathfrak{M}_{0}$.

Published by the American Physical Society under the terms of the Creative Commons Attribution 4.0 International license. Further distribution of this work must maintain attribution to the author(s) and the published article's title, journal citation, and DOI. Funded by SCOAP ${ }^{3}$.
}

$M_{\pi}=140 \mathrm{MeV}$, the entropy density also drops in the presence of an external magnetic field whose impact is most distinct around the temperature $T \approx 40 \mathrm{MeV}$. While the results for the dependence of entropy density on magnetic field strength, temperature, and arbitrary pion mass are new to the best of our knowledge, the magnetic-field induced decrease of the entropy density at the physical point $M_{\pi}=140 \mathrm{MeV}$ has also been observed in the hadron resonance gas model [13], and in the $(2+1)$ flavor Polyakov-loop quark-meson model [21]. However, the comparison is only qualitative because the latter reference is based on three flavors, and the hadron resonance gas model includes even more particles. But most importantly, our CHPT study is fully systematic and model independent.

The magnetization induced at finite temperature is negative in the entire parameter region accessible by CHPT $\left(T, M_{\pi}, \sqrt{|q H|} \lesssim 0.2 \mathrm{GeV}\right)$, which includes the physically most relevant case $M_{\pi}=140 \mathrm{MeV}$. The magnitude of the finite-temperature magnetization grows as both magnetic field strength and temperature increase, implying that the $\mathrm{QCD}$ vacuum behaves as a diamagnetic medium at low temperatures and weak magnetic fields. This is fully consistent with conclusions drawn from lattice QCD [3,27-29], the $(2+1)$ flavor Polyakov-loop quarkmeson model [21], and the three-flavor quark-meson model with $U_{A}(1)$ anomaly [30]. Still, the region accessible by CHPT has not been fully addressed in these references.

Finally we point out that the characteristics of the entropy density and the finite-temperature quark condensate in a magnetic field are correlated. This becomes most transparent when the $H=0$ portions in either quantity are subtracted to unmask the effect of the magnetic field. We observe that for arbitrary pion masses-including the physical point $M_{\pi}=140 \mathrm{MeV}$ - the enhancement of the finite-temperature quark condensate in a magnetic field is reflected in a decrease of the entropy density. 
The article is organized as follows. To set the stage for our discussion, in Sec. II we provide the two-loop representation for the free energy density and explain our notation. In Sec. III we derive the entropy density and explore its dependence on temperature, magnetic field strength and arbitrary pion masses, including the physical point $M_{\pi}=140 \mathrm{MeV}$. The two-loop representation for the magnetization induced at finite temperature is derived in Sec. IV and its properties in magnetic fields and for arbitrary pion masses is elucidated in various figures. Section $\mathrm{V}$ is devoted to the connection between order parameter and entropy density. Finally, Sec. VI contains our conclusions.

\section{PRELIMINARIES}

Two-flavor chiral perturbation theory ${ }^{2}$ subjected to a magnetic background $H$ and at finite temperature has been used by various authors to explore the low-energy regime of quantum chromodynamics [24-26,36-45]. The starting point of the present study is the two-loop representation for the free energy density derived in Ref. [25], ${ }^{3}$

$$
z=z_{0}+z^{T} .
$$

Here $z_{0}$ is the vacuum energy density (free energy density at $T=0)$ and $z^{T}$ represents the finite-temperature portion. The latter amounts to

$$
\begin{aligned}
z^{T}= & -g_{0}\left(M_{\pi}^{ \pm}, T, 0\right)-\frac{1}{2} g_{0}\left(M_{\pi}^{0}, T, 0\right)-\tilde{g}_{0}\left(M_{\pi}^{ \pm}, T, H\right) \\
& +\frac{M_{\pi}^{2}}{2 F^{2}} g_{1}\left(M_{\pi}^{ \pm}, T, 0\right) g_{1}\left(M_{\pi}^{0}, T, 0\right)-\frac{M_{\pi}^{2}}{8 F^{2}}\left\{g_{1}\left(M_{\pi}^{0}, T, 0\right)\right\}^{2} \\
& +\frac{M_{\pi}^{2}}{2 F^{2}} g_{1}\left(M_{\pi}^{0}, T, 0\right) \tilde{g}_{1}\left(M_{\pi}^{ \pm}, T, H\right)+\mathcal{O}\left(p^{8}\right)
\end{aligned}
$$

and relies on the kinematical Bose functions

$$
\begin{aligned}
g_{0}(\mathcal{M}, T, 0)= & T^{4} \int_{0}^{\infty} \mathrm{d} \rho \rho^{-3} \exp \left(-\frac{\mathcal{M}^{2}}{4 \pi T^{2}} \rho\right)\left[S\left(\frac{1}{\rho}\right)-1\right], \\
g_{1}(\mathcal{M}, T, 0)= & \frac{T^{2}}{4 \pi} \int_{0}^{\infty} \mathrm{d} \rho \rho^{-2} \exp \left(-\frac{\mathcal{M}^{2}}{4 \pi T^{2}} \rho\right)\left[S\left(\frac{1}{\rho}\right)-1\right], \\
\tilde{g}_{0}\left(M_{\pi}^{ \pm}, T, H\right)= & \frac{T^{2}}{4 \pi}|q H| \int_{0}^{\infty} \mathrm{d} \rho \rho^{-2}\left(\frac{1}{\sinh \left(|q H| \rho / 4 \pi T^{2}\right)}-\frac{4 \pi T^{2}}{|q H| \rho}\right) \\
& \times \exp \left(-\frac{\left(M_{\pi}^{ \pm}\right)^{2}}{4 \pi T^{2}} \rho\right)\left[S\left(\frac{1}{\rho}\right)-1\right], \\
\tilde{g}_{1}\left(M_{\pi}^{ \pm}, T, H\right)= & \frac{1}{16 \pi^{2}}|q H| \int_{0}^{\infty} \mathrm{d} \rho \rho^{-1}\left(\frac{1}{\sinh \left(|q H| \rho / 4 \pi T^{2}\right)}-\frac{4 \pi T^{2}}{|q H| \rho}\right) \\
& \times \exp \left(-\frac{\left(M_{\pi}^{ \pm}\right)^{2}}{4 \pi T^{2}} \rho\right)\left[S\left(\frac{1}{\rho}\right)-1\right],
\end{aligned}
$$

with $S(z)$,

$$
S(z)=\sum_{n=-\infty}^{\infty} \exp \left(-\pi n^{2} z\right)
$$

as the Jacobi theta function. These kinematical Bose functions depend on the masses of the charged $\left(M_{\pi}^{ \pm}\right)$ and neutral $\left(M_{\pi}^{0}\right)$ pions in a magnetic field, namely,

\footnotetext{
${ }^{2}$ Introductions to chiral perturbation theory are given in Refs. [31-35].

${ }^{3}$ We confine ourselves to the isospin limit $m_{u}=m_{d}$.
}

$$
\begin{aligned}
& \left(M_{\pi}^{ \pm}\right)^{2}=M_{\pi}^{2}+\frac{\bar{l}_{6}-\bar{l}_{5}}{48 \pi^{2}} \frac{|q H|^{2}}{F^{2}}, \\
& \left(M_{\pi}^{0}\right)^{2}=M_{\pi}^{2}+\frac{M^{2}}{F^{2}} K_{1},
\end{aligned}
$$

where $K_{1}$ corresponds to the integral

$$
K_{1}=\frac{|q H|}{16 \pi^{2}} \int_{0}^{\infty} \mathrm{d} \rho \rho^{-1} \exp \left(-\frac{M_{\pi}^{2}}{|q H|} \rho\right)\left(\frac{1}{\sinh (\rho)}-\frac{1}{\rho}\right) \text {, }
$$

$q$ is the electric charge, and $\bar{l}_{5}, \bar{l}_{6}$ are renormalized nextto-leading order (NLO) low-energy effective constants. 
The mass $\mathcal{M}$ appearing in the kinematical Bose functions $g_{0}$ and $g_{1}$ can either stand for $M_{\pi}^{ \pm}$or $\boldsymbol{M}_{\pi}^{0}$. Finally, the mass $M_{\pi}$ is the renormalized pion mass in zero magnetic field,

$$
M_{\pi}^{2}=M^{2}-\frac{\bar{l}_{3}}{32 \pi^{2}} \frac{M^{4}}{F^{2}}+\mathcal{O}\left(M^{6}\right)
$$

where $M(F)$ is the tree-level pion mass (pion decay constant).

\section{ENTROPY DENSITY}

In the previous section we have defined the kinematical functions $g_{r}$ and $\tilde{g}_{r}$ that are dimensionful. In what follows it is more convenient to use the dimensionless functions $h_{r}$ and $\tilde{h}_{r}$,

$$
\begin{array}{lll}
h_{0}=\frac{g_{0}}{T^{4}}, & \tilde{h}_{0}=\frac{\tilde{g}_{0}}{T^{4}}, & h_{1}=\frac{g_{1}}{T^{2}}, \\
\tilde{h}_{1}=\frac{\tilde{g}_{1}}{T^{2}}, & h_{2}=g_{2}, & \tilde{h}_{2}=\tilde{g}_{2} .
\end{array}
$$

In addition, instead of using absolute values of temperature, pion mass, and magnetic field strength, we prefer to work with the normalized and dimensionless quantities $t, m$, and $m_{H}$ defined as

$$
t=\frac{T}{4 \pi F}, \quad m=\frac{M_{\pi}}{4 \pi F}, \quad m_{H}=\frac{\sqrt{|q H|}}{4 \pi F} .
$$

The common denominator represents the chiral symmetry breaking scale $\Lambda_{\chi} \approx 4 \pi F \approx 1 \mathrm{GeV}$. In the low-energy region where chiral perturbation theory operates, the parameters $t, m$, and $m_{H}$ are small. In subsequent plots, the value of the (tree-level) pion decay constant is $F=$ 85.6 MeV (see Ref. [46]).

The entropy density $s$ can be extracted from the pressure via

$$
s=\frac{\mathrm{d} P}{\mathrm{~d} T} .
$$

The pressure in a homogeneous medium, up to the sign, is nothing but the finite-temperature piece in the free energy density,

$$
P=-z^{T}
$$

Derivatives of the Bose functions $g_{r}$ and $\tilde{g}_{r}$ with respect to temperature are easily obtained with the relations

$$
\begin{aligned}
\frac{\mathrm{d}}{\mathrm{d} T} g_{r}(\mathcal{M}, T, 0)= & \frac{2 \mathcal{M}^{2}}{T} g_{r+1}(\mathcal{M}, T, 0)+\frac{d-2 r}{T} g_{r}(\mathcal{M}, T, 0), \\
\frac{\mathrm{d}}{\mathrm{d} T} \tilde{g}_{r}\left(M_{\pi}^{ \pm}, T, H\right)= & \frac{2 M^{2}}{T} \tilde{g}_{r+1}\left(M_{\pi}^{ \pm}, T, H\right)+\frac{d-2 r-2}{T} \tilde{g}_{r}\left(M_{\pi}^{ \pm}, T, H\right) \\
& +\tilde{g}_{r}^{[1]}\left(M_{\pi}^{ \pm}, T, H\right),
\end{aligned}
$$

where

$$
\begin{aligned}
\tilde{g}_{r}^{[1]}\left(M_{\pi}^{ \pm}, T, H\right)= & \frac{T^{d-2 r-2}}{(4 \pi)^{r+1}}|q H| \int_{0}^{\infty} \mathrm{d} \rho \rho^{r-\frac{d}{2}}\left(\frac{|q H| \rho \operatorname{coth}\left(|q H| \rho / 4 \pi T^{2}\right)}{2 \pi T^{3} \sinh \left(|q H| \rho / 4 \pi T^{2}\right)}-\frac{8 \pi T}{|q H| \rho}\right) \\
& \times \exp \left(-\frac{\left(M_{\pi}^{ \pm}\right)^{2}}{4 \pi T^{2}} \rho\right)\left[S\left(\frac{1}{\rho}\right)-1\right] .
\end{aligned}
$$

On the basis of the representation for $z^{T}$, Eq. (2.2), the two-loop entropy density—scaled by $1 / T^{3}$ —amounts to

$$
\begin{aligned}
\frac{s}{T^{3}}= & 2 s_{ \pm} h_{1}\left(M_{\pi}^{ \pm}, T, 0\right)+4 h_{0}\left(M_{\pi}^{ \pm}, T, 0\right)+s_{0} h_{1}\left(M_{\pi}^{0}, T, 0\right)+2 h_{0}\left(M_{\pi}^{0}, T, 0\right) \\
& +2 s_{ \pm} \tilde{h}_{1}\left(M_{\pi}^{ \pm}, T, H\right)+2 \tilde{h}_{0}\left(M_{\pi}^{ \pm}, T, H\right)+\tilde{h}_{0}^{[1]}\left(M_{\pi}^{ \pm}, T, H\right) \\
& -8 \pi^{2} m^{2}\left\{2 s_{ \pm} h_{2}\left(M_{\pi}^{ \pm}, T, 0\right) h_{1}\left(M_{\pi}^{0}, T, 0\right)+4 h_{1}\left(M_{\pi}^{ \pm}, T, 0\right) h_{1}\left(M_{\pi}^{0}, T, 0\right)\right. \\
& +2 s_{0} h_{1}\left(M_{\pi}^{ \pm}, T, 0\right) h_{2}\left(M_{\pi}^{0}, T, 0\right)+2 s_{0} h_{2}\left(M_{\pi}^{0}, T, 0\right) \tilde{h}_{1}\left(M_{\pi}^{ \pm}, T, H\right) \\
& +2 h_{1}\left(M_{\pi}^{0}, T, 0\right) \tilde{h}_{1}\left(M_{\pi}^{ \pm}, T, H\right)+2 s_{ \pm} h_{1}\left(M_{\pi}^{0}, T, 0\right) \tilde{h}_{2}\left(M_{\pi}^{ \pm}, T, H\right) \\
& +h_{1}\left(M_{\pi}^{0}, T, 0\right) \tilde{h}_{1}^{[1]}\left(M_{\pi}^{ \pm}, T, H\right)-s_{0} h_{1}\left(M_{\pi}^{0}, T, 0\right) h_{2}\left(M_{\pi}^{0}, T, 0\right) \\
& \left.-h_{1}\left(M_{\pi}^{0}, T, 0\right) h_{1}\left(M_{\pi}^{0}, T, 0\right)\right\},
\end{aligned}
$$



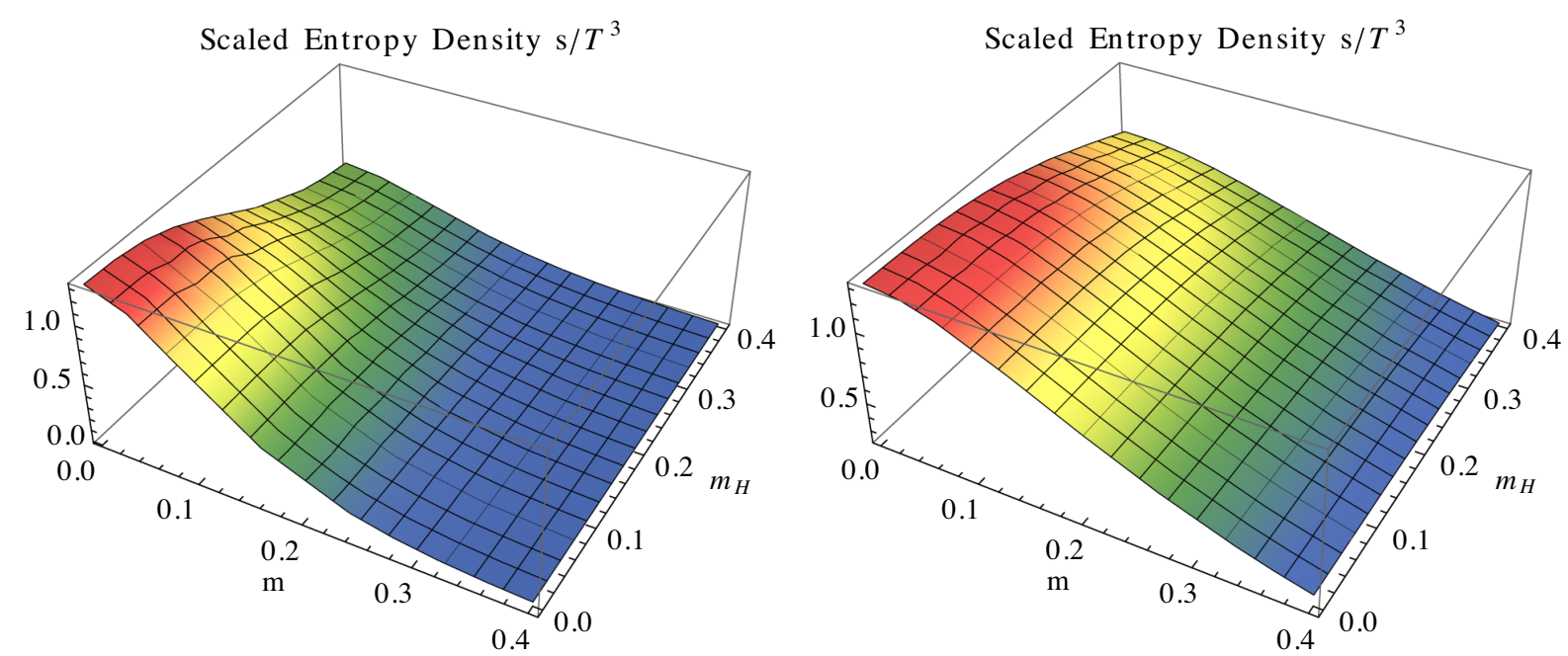

FIG. 1. Scaled entropy density $s / T^{3}$ : Sum of one- and two-loop contributions for $T=54 \mathrm{MeV}$ (left) and $T=108 \mathrm{MeV}$ (right) in terms of the dimensionless parameters $m, m_{H}$.

with coefficients

$$
\begin{aligned}
& s_{ \pm}=\frac{m^{2}}{t^{2}}+\frac{\left(\bar{l}_{6}-\bar{l}_{5}\right) m_{H}^{4}}{3 t^{2}} \\
& s_{0}=\frac{m^{2}}{t^{2}}+\frac{m^{2} m_{H}^{2}}{t^{2}} \int_{0}^{\infty} \mathrm{d} \rho \rho^{-1} \exp \left(-\frac{m^{2}}{m_{H}^{2}} \rho\right)\left(\frac{1}{\sinh (\rho)}-\frac{1}{\rho}\right) .
\end{aligned}
$$

The dimensionless functions $\tilde{h}_{0}^{[1]}\left(M_{\pi}^{ \pm}, T, H\right)$ and $\tilde{h}_{1}^{[1]}\left(M_{\pi}^{ \pm}, T, H\right)$ are

$$
\tilde{h}_{0}^{[1]}\left(M_{\pi}^{ \pm}, T, H\right)=\frac{\tilde{g}_{0}^{[1]}\left(M_{\pi}^{ \pm}, T, H\right)}{T^{3}}, \quad \tilde{h}_{1}^{[1]}\left(M_{\pi}^{ \pm}, T, H\right)=\frac{\tilde{g}_{1}^{[1]}\left(M_{\pi}^{ \pm}, T, H\right)}{T}
$$

In Fig. 1 we plot the scaled entropy density $s / T^{3}$ - sum of one- and two-loop contributions given in Eq. (3.7) - in terms of magnetic field strength $\left(m_{H}\right)$ and pion mass $(m)$ at the fixed temperatures $T=\{54,108\} \mathrm{MeV}(t=\{0.05,0.1\})$. The entropy density decreases when the magnetic field becomes stronger and it also decreases when the masses of the pions grow. The impact of the pion mass, however, is more pronounced than the dependence on the magnetic field. The limits $M \rightarrow 0$ (chiral limit) and $H \rightarrow 0$ do not pose any problems. In particular, taking the double limit $\{M, H\} \rightarrow 0$, the scaled entropy density tends to the value describing the noninteracting Bose gas,

$$
\frac{s(T, 0,0)}{T^{3}}=\frac{2 \pi^{2}}{15} \approx 1.32
$$

At first sight it may be surprising that the CHPT representation of the entropy density in this double limit reduces to the noninteracting — and not the "weakly" interactingBose gas. The crucial point is that in the chiral perturbation theory expansion, the pion-pion interaction only starts manifesting itself beyond two loop order in the double limit $\{M, H\} \rightarrow 0 .{ }^{4}$ In the pioneering three-loop CHPT evaluation of the QCD partition function in zero magnetic field, Ref. [47], it was demonstrated that the interaction in the entropy density-much like in the pressure, internal energy, and heat capacity-only starts showing up at the three-loop level in the chiral limit. In this perspective, the pion-pion interaction indeed is very weak. It should be mentioned that a three-loop evaluation of the QCD partition function in nonzero magnetic field-although feasible within chiral perturbation theory-is technically extremely demanding. Still, respective work is in progress [48].

To better assess the effect of the magnetic field on entropy, we subtract the $H=0$ contribution, i.e., consider the quantity

$$
\frac{s_{H}}{T^{3}}=\frac{s\left(T, M_{\pi}, H\right)-s\left(T, M_{\pi}, 0\right)}{T^{3}}
$$

\footnotetext{
${ }^{4}$ Recall that the present CHPT analysis in nonzero magnetic field refers to two-loop order.
} 
Scaled Subtracted Entropy Density $s_{H} / T^{3}$ 0.0

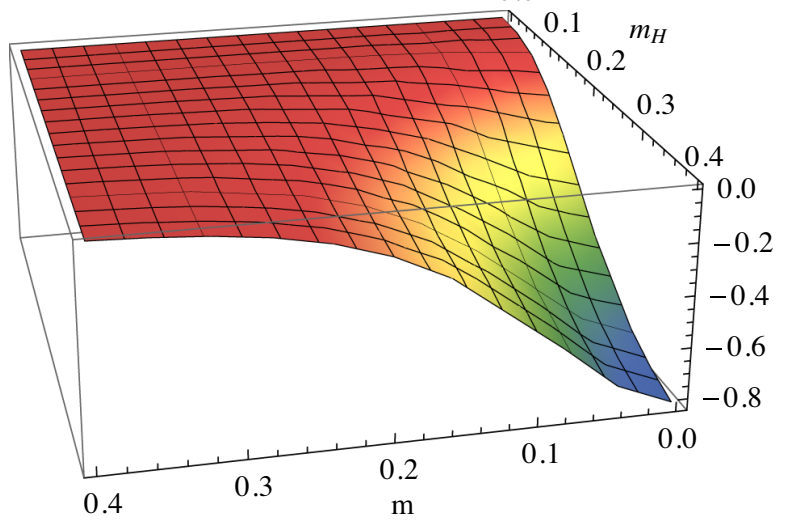

Scaled Subtracted Entropy Density $s_{H} / T^{3}$

0.0

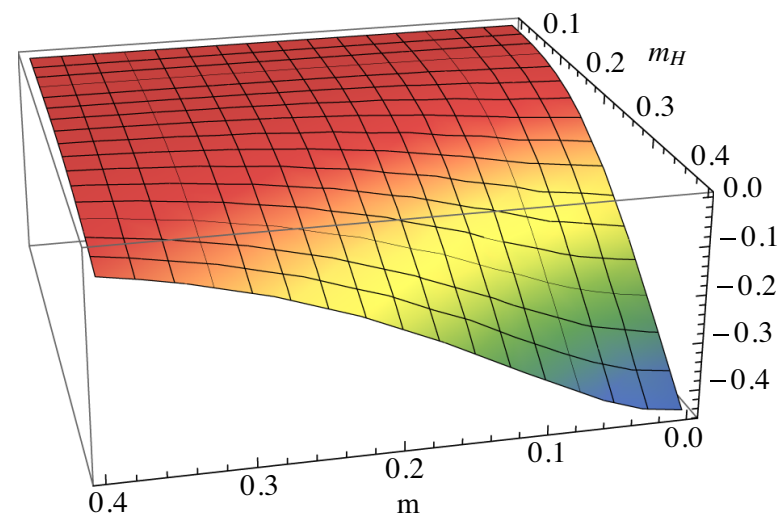

FIG. 2. Subtracted scaled entropy density $s_{H} / T^{3}$ : Sum of one- and two-loop contributions for $T=54 \mathrm{MeV}$ (left) and $T=108 \mathrm{MeV}$ (right) in terms of the dimensionless parameters $m, m_{H}$.

that measures the influence of the magnetic field. In Fig. 2 we plot $s_{H} / T^{3}$ in terms of magnetic field strength $\left(m_{H}\right)$ and pion mass $(m)$ at the same fixed temperatures $T=\{54,108\} \mathrm{MeV}$. Overall, in presence of the magnetic field, the entropy density drops. For a given fixed $m_{H}$, the effect becomes most pronounced when the chiral limit is approached.

Let us now focus on the real world defined by the physical point $M_{\pi}=140 \mathrm{MeV}(m=0.130)$. In Fig. 3, on the left-hand side (lhs), we plot the scaled two-loop entropy density $s / T^{3}$ according to Eq. (3.7) in function of magnetic field strength $\left(m_{H}\right)$ and temperature $(t)$. Clearly, the impact of temperature is predominant and the slight decrease of the entropy density caused by the magnetic field is hardly visible. Therefore, we rather consider the quantity $s_{H} / T^{3}$ defined by Eq. (3.11) that isolates the effect of the magnetic field. Indeed, as illustrated on the right-hand side (rhs) of
Fig. 3, the presence of the magnetic field lowers the entropy density in an interesting and nontrivial way: the effect is most pronounced around $t \approx 0.035$, corresponding to $T \approx$ $40 \mathrm{MeV}$. As we discuss below, the increase of order-as witnessed by the drop in entropy density-is reflected in the behavior of the finite-temperature quark condensate.

While our results regarding the dependence of entropy density on magnetic field strength, temperature, and arbitrary pion mass are new to the best of our knowledge, the behavior of the entropy density at the physical pion mass has been explored in the hadron resonance gas model [13], as well as in the $(2+1)$ flavor Polyakov-loop quark-meson model [21]. Indeed, both references also report a decrease of entropy density caused by the magnetic field in the relevant region $T \lesssim 100 \mathrm{MeV}$ that is accessible with chiral perturbation theory. The comparison, however, is only qualitative as the latter reference is based on three flavors
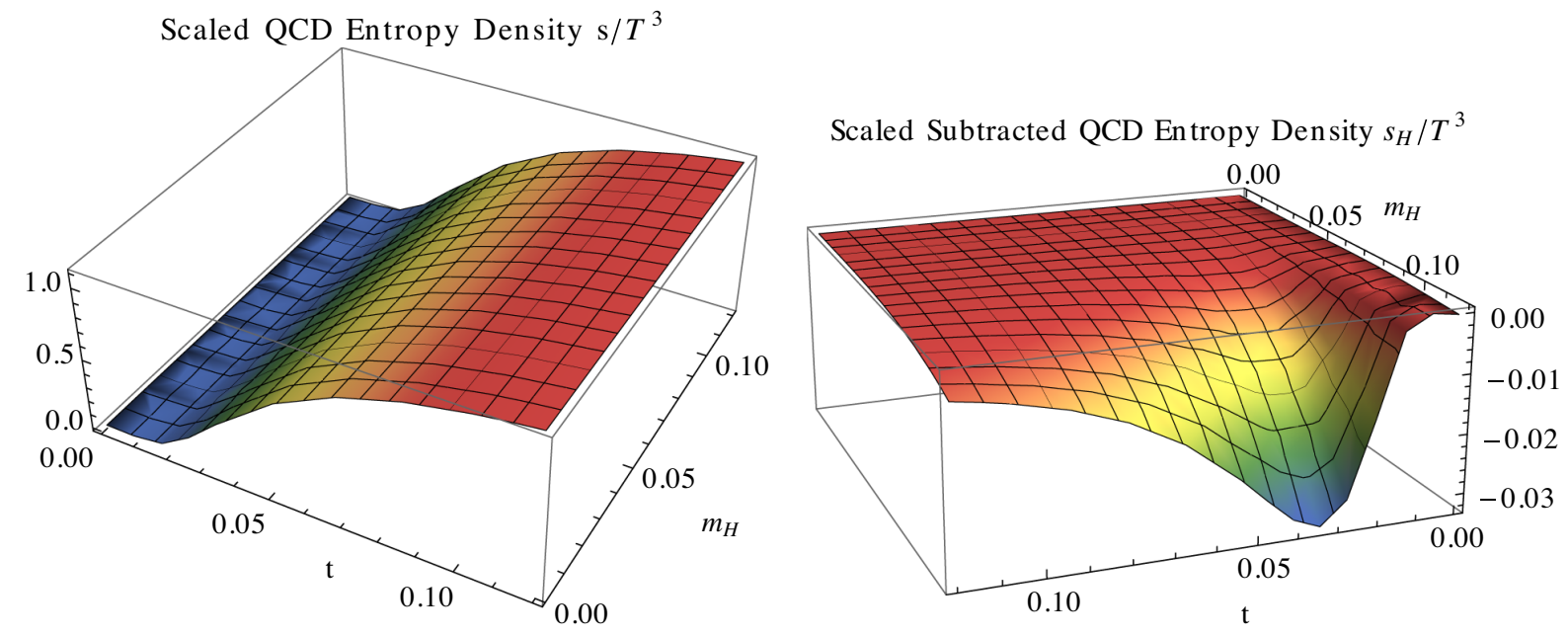

FIG. 3. Scaled QCD entropy density $s / T^{3}$ (lhs) and scaled subtracted QCD entropy density $s_{H} / T^{3}$ (rhs). Both quantities in terms of the dimensionless parameters $m_{H}$ (magnetic field strength) and $t$ (temperature). 
and the hadron resonance gas model also includes more particles than just the three pions.

\section{FINITE-TEMPERATURE MAGNETIZATION}

The total magnetization $\mathfrak{M}_{\text {tot }}$ is defined as the (negative) derivative of the free energy density, Eq. (2.1), with respect to the magnetic field,

$$
\mathfrak{M}_{\text {tot }}(T, M, H)=-\frac{\mathrm{d} z}{\mathrm{~d}|q H|}
$$

Notice that the total magnetization $\mathfrak{M}_{\text {tot }}$ can be decomposed into a temperature-dependent piece $\mathfrak{M}_{T}$ and temperature-independent piece $\mathfrak{M}_{0}$,

$$
\mathfrak{M}_{\text {tot }}=\mathfrak{M}_{T}+\mathfrak{M}_{0}
$$

In this study we focus on the former. ${ }^{5}$

The finite-temperature free energy density $z^{T}$, Eq. (2.2), involves various types of kinematical Bose functions. Corresponding derivatives with respect to the magnetic field are readily obtained via

$$
\begin{aligned}
\frac{\mathrm{d}}{\mathrm{d}|q H|} g_{r}\left(M_{\pi}^{ \pm}, T, 0\right) & =-\frac{\bar{l}_{6}-\bar{l}_{5}}{24 \pi^{2}} \frac{|q H|}{F^{2}} g_{r+1}\left(M_{\pi}^{ \pm}, T, 0\right), \\
\frac{\mathrm{d}}{\mathrm{d}|q H|} g_{r}\left(M_{\pi}^{0}, T, 0\right) & =-\frac{M_{\pi}^{2}}{F^{2}} \frac{\mathrm{d} K_{1}}{\mathrm{~d}|q H|} g_{r+1}\left(M_{\pi}^{0}, T, 0\right), \\
\frac{\mathrm{d}}{\mathrm{d}|q H|} \tilde{g}_{r}\left(M_{\pi}^{ \pm}, T, H\right) & =\frac{1}{|q H|} \tilde{g}_{r}\left(M_{\pi}^{ \pm}, T, H\right)-\frac{\bar{l}_{6}-\bar{l}_{5}}{24 \pi^{2}} \frac{|q H|}{F^{2}} \tilde{g}_{r+1}\left(M_{\pi}^{ \pm}, T, H\right)+\tilde{g}_{r}^{[H]}\left(M_{\pi}^{ \pm}, T, H\right),
\end{aligned}
$$

where

$$
\begin{aligned}
\tilde{g}_{r}^{[H]}\left(M_{\pi}^{ \pm}, T, H\right)= & \frac{T^{d-2 r-2}}{(4 \pi)^{r+1}}|q H| \int_{0}^{\infty} \mathrm{d} \rho \rho^{r-\frac{d}{2}}\left(-\frac{\rho \operatorname{coth}\left(|q H| \rho / 4 \pi T^{2}\right)}{4 \pi T^{2} \sinh \left(|q H| \rho / 4 \pi T^{2}\right)}+\frac{4 \pi T^{2}}{|q H|^{2} \rho}\right) \\
& \times \exp \left(-\frac{\left(M_{\pi}^{ \pm}\right)^{2}}{4 \pi T^{2}} \rho\right)\left[S\left(\frac{1}{\rho}\right)-1\right] .
\end{aligned}
$$

The finite-temperature magnetization $\mathfrak{M}_{T}(T, M, H)$ —up to two loops and scaled by $1 / T^{2}$ —then takes the form

$$
\begin{aligned}
\frac{\mathfrak{M}_{T}}{T^{2}}= & m_{ \pm} h_{1}\left(M_{\pi}^{ \pm}, T, 0\right)+\frac{m_{0}}{2} h_{1}\left(M_{\pi}^{0}, T, 0\right)+\frac{t^{2}}{m_{H}^{2}} \tilde{h}_{0}\left(M_{\pi}^{ \pm}, T, H\right) \\
& +m_{ \pm} \tilde{h}_{1}\left(M_{\pi}^{ \pm}, T, H\right)+\tilde{h}_{0}^{[H]}\left(M_{\pi}^{ \pm}, T, H\right) \\
& -4 \pi^{2} m^{2}\left\{2 m_{ \pm} h_{2}\left(M_{\pi}^{ \pm}, T, 0\right) h_{1}\left(M_{\pi}^{0}, T, 0\right)+2 m_{0} h_{1}\left(M_{\pi}^{ \pm}, T, 0\right) h_{2}\left(M_{\pi}^{0}, T, 0\right)\right. \\
& +2 m_{0} h_{2}\left(M_{\pi}^{0}, T, 0\right) \tilde{h}_{1}\left(M_{\pi}^{ \pm}, T, H\right)+\frac{2 t^{2}}{m_{H}^{2}} h_{1}\left(M_{\pi}^{0}, T, 0\right) \tilde{h}_{1}\left(M_{\pi}^{ \pm}, T, H\right) \\
& +2 m_{ \pm} h_{1}\left(M_{\pi}^{0}, T, 0\right) \tilde{h}_{2}\left(M_{\pi}^{ \pm}, T, H\right)+2 h_{1}\left(M_{\pi}^{0}, T, 0\right) \tilde{h}_{1}^{[H]}\left(M_{\pi}^{ \pm}, T, H\right) \\
& \left.-m_{0} h_{2}\left(M_{\pi}^{0}, T, 0\right) h_{1}\left(M_{\pi}^{0}, T, 0\right)\right\}
\end{aligned}
$$

with coefficients

$$
\begin{aligned}
& m_{ \pm}=-\frac{2\left(\bar{l}_{6}-\bar{l}_{5}\right) m_{H}^{2}}{3} \\
& m_{0}=-m^{2} \int_{0}^{\infty} \mathrm{d} \rho \rho^{-1} \exp \left(-\frac{m^{2}}{m_{H}^{2}} \rho\right)\left(\frac{1}{\sinh (\rho)}-\frac{1}{\rho}\right)-\frac{m^{4}}{m_{H}^{2}} \int_{0}^{\infty} \mathrm{d} \rho \exp \left(-\frac{m^{2}}{m_{H}^{2}} \rho\right)\left(\frac{1}{\sinh (\rho)}-\frac{1}{\rho}\right) .
\end{aligned}
$$

\footnotetext{
${ }^{5}$ The chiral perturbation theory representation for the zero-temperature magnetization $\mathfrak{M}_{0}$ is derived in the companion paper [49]. That reference also contains a detailed discussion of the total magnetization $\mathfrak{M}_{\text {tot }}$ and the total magnetic susceptibility $\chi_{\text {tot }}=\chi_{T}+\chi_{0}$, as well as an analysis of the associated diamagnetic and paramagnetic phases.
} 

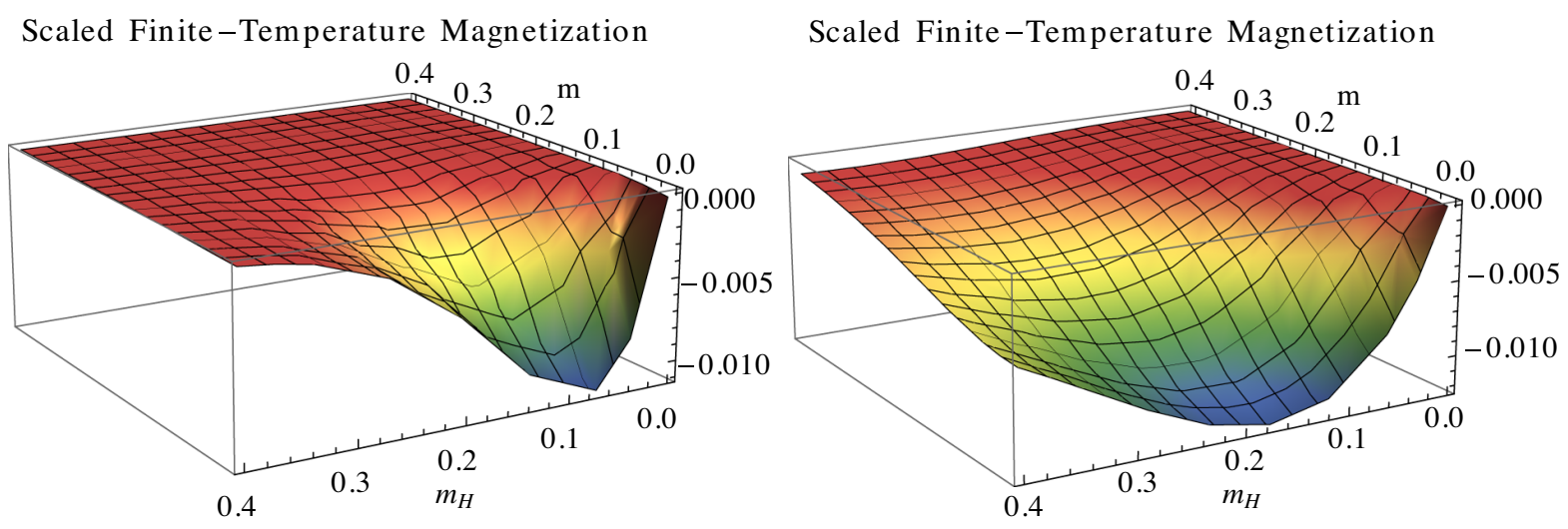

FIG. 4. Scaled finite-temperature magnetization $\mathfrak{M}_{T} / T^{2}$ : Sum of one- and two-loop contributions for $T=54 \mathrm{MeV}$ (left) and $T=108 \mathrm{MeV}$ (right) in terms of the dimensionless parameters $m, m_{H}$.

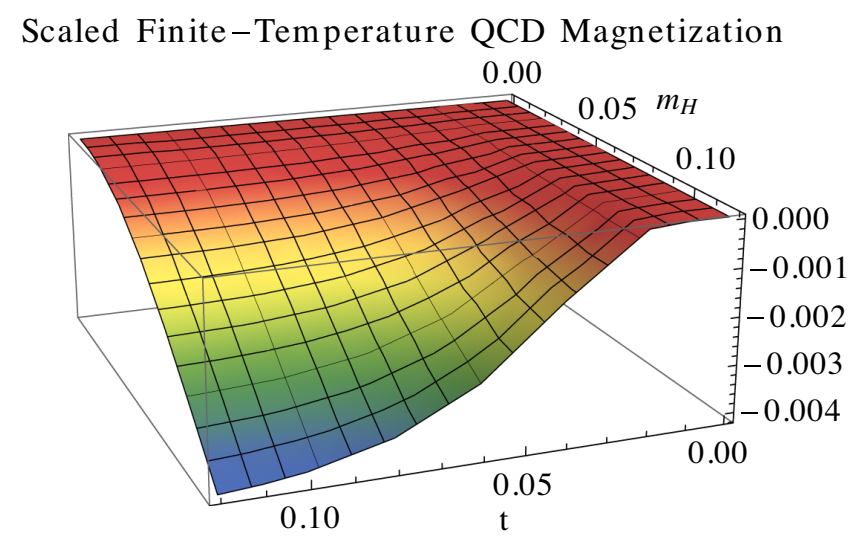

FIG. 5. Scaled finite-temperature QCD magnetization $\mathfrak{M}_{T} / T^{2}$ in terms of the dimensionless parameters $m_{H}$ (magnetic field strength) and $t$ (temperature).

The dimensionless functions $\tilde{h}_{0}^{[H]}\left(M_{\pi}^{ \pm}, T, H\right)$ and $\tilde{h}_{1}^{[H]}\left(M_{\pi}^{ \pm}, T, H\right)$ are

$$
\begin{aligned}
& \tilde{h}_{0}^{[H]}\left(M_{\pi}^{ \pm}, T, H\right)=\frac{\tilde{g}_{0}^{[H]}\left(M_{\pi}^{ \pm}, T, H\right)}{T^{2}}, \\
& \tilde{h}_{1}^{[H]}\left(M_{\pi}^{ \pm}, T, H\right)=\tilde{g}_{1}^{[H]}\left(M_{\pi}^{ \pm}, T, H\right) .
\end{aligned}
$$

In Fig. 4 we plot $\mathfrak{M}_{T} / T^{2}$ in terms of magnetic field strength $\left(m_{H}\right)$ and pion mass $(m)$ at the fixed temperatures $T=\{54,108\} \mathrm{MeV}(t=\{0.05,0.1\})$. The finitetemperature magnetization turns out to be negative in the entire parameter region under consideration. ${ }^{6}$ Note that its dependence on magnetic field strength is nontrivial: remarkably, the magnitude of the finite-temperature magnetization initially grows as the magnetic field

\footnotetext{
${ }^{6}$ Recall that the finite-temperature magnetization $\mathfrak{M}_{T}$ corresponds to the total magnetization with the zero-temperature portion $\mathfrak{M}_{0}$ subtracted: $\mathfrak{M}_{T}=\mathfrak{M}_{\text {tot }}-\mathfrak{M}_{0}$. The finite-temperature magnetization $\mathfrak{M}_{T}$ thus measures the change in the magnetization when temperature is raised from $T=0$ to $T \neq 0$.
}

increases, but then declines in stronger magnetic fields. For fixed magnetic field strength, the effect becomes more pronounced as one approaches the chiral limit. On the other hand, the limit $H \rightarrow 0$ is trivial: the finite-temperature magnetization simply vanishes as it should. Accordingly, there is no need to subtract the $H=0$ contribution (as for the entropy density): the quantity $\mathfrak{M}_{T}(T, M, H)$ already measures the impact of the magnetic field.

In Fig. 5 we explore the dependence of $\mathfrak{M}_{T} / T^{2}$ on magnetic field strength and temperature in the real world with physical pion mass $M_{\pi}=140 \mathrm{MeV}(m=0.130)$. The induced finite-temperature magnetization is negative and its magnitude grows as both magnetic field and temperature increase. The dependence of $\mathfrak{M}_{T} / T^{2}$ on the magnetic field - at fixed temperature-implies that the QCD vacuum behaves as a diamagnetic medium at low temperatures and in weak magnetic fields.

This conclusion fully agrees with studies based on lattice QCD [3,27-29], on the $(2+1)$ flavor Polyakov-loop quark-meson model [21], and on the three-flavor quarkmeson model with $U_{A}(1)$ anomaly [30]. In these references the finite-temperature magnetic susceptibility $\chi_{B}(T)$,

$$
\chi_{B}(T)=\lim _{H \rightarrow 0}-\frac{\mathrm{d}^{2}}{\mathrm{~d}|q H|^{2}}\left\{z-z_{0}\right\}
$$

was considered. At lower temperatures where only pions are the relevant degrees of freedom, the authors indeed find that the magnetic susceptibility is negative. In particular, its value at $T=90 \mathrm{MeV}$, reported in Ref. [3],

$$
\chi_{B}(T=90 \mathrm{MeV})=-0.002(2),
$$

is perfectly consistent with our two-loop CHPT analysis where we extract ${ }^{7}$

$$
\chi_{B}(T=90 \mathrm{MeV})=-0.00245 .
$$

\footnotetext{
${ }^{7}$ We have used $F=85.6 \mathrm{MeV}$ from Ref. [46] and-in accordance with Ref. [13] $-M_{\pi}=135 \mathrm{MeV}$.
} 


\section{FINITE-TEMPERATURE QUARK CONDENSATE}

It is illuminating to include the quark condensate $\langle\bar{q} q\rangle$ into our discussion and compare its properties at low temperatures and weak magnetic fields with the behavior of the entropy density. The two-loop representation for the quark condensate, decomposed into $T=0$ and finite-temperature contributions,

$$
\langle\bar{q} q\rangle=\langle 0|\bar{q} q| 0\rangle+\langle\bar{q} q\rangle^{T},
$$

has been derived in Ref. [26]. Here we just quote the result for the finite-temperature portion $\langle\bar{q} q\rangle^{T}$ that is relevant to make our point, ${ }^{8}$

$$
\frac{\langle\bar{q} q\rangle^{T}}{\langle 0|\bar{q} q| 0\rangle_{0}}\left(1-\frac{M_{\pi}^{2}}{32 \pi^{2} F^{2}}\left(2 \bar{l}_{3}-1\right)\right)^{-1}=-\left\{\frac{q_{1}}{F^{2}} T^{2}+\frac{q_{2}}{F^{4}} T^{4}+\mathcal{O}\left(T^{6}\right)\right\}
$$

with coefficients

$$
\begin{aligned}
q_{1}= & h_{1}\left(M_{\pi}^{ \pm}, T, 0\right)+\frac{1}{2} a_{0} h_{1}\left(M_{\pi}^{0}, T, 0\right)+\tilde{h}_{1}\left(M_{\pi}^{ \pm}, T, H\right), \\
q_{2}= & +\frac{1}{2} h_{1}\left(M_{\pi}^{ \pm}, T, 0\right) h_{1}\left(M_{\pi}^{0}, T, 0\right)+\frac{1}{2} h_{1}\left(M_{\pi}^{0}, T, 0\right) \tilde{h}_{1}\left(M_{\pi}^{ \pm}, T, H\right) \\
& -\frac{1}{8} h_{1}\left(M_{\pi}^{0}, T, 0\right) h_{1}\left(M_{\pi}^{0}, T, 0\right)-\frac{1}{2} \frac{m^{2}}{t^{2}} h_{1}\left(M_{\pi}^{0}, T, 0\right) h_{2}\left(M_{\pi}^{ \pm}, T, 0\right) \\
& -\frac{1}{2} a_{0} \frac{m^{2}}{t^{2}} h_{1}\left(M_{\pi}^{ \pm}, T, 0\right) h_{2}\left(M_{\pi}^{0}, T, 0\right)-\frac{1}{2} a_{0} \frac{m^{2}}{t^{2}} \tilde{h}_{1}\left(M_{\pi}^{ \pm}, T, H\right) h_{2}\left(M_{\pi}^{0}, T, 0\right) \\
& +\frac{1}{4} a_{0} \frac{m^{2}}{t^{2}} h_{1}\left(M_{\pi}^{0}, T, 0\right) h_{2}\left(M_{\pi}^{0}, T, 0\right)-\frac{1}{2} \frac{m^{2}}{t^{2}} h_{1}\left(M_{\pi}^{0}, T, 0\right) \tilde{h}_{2}\left(M_{\pi}^{ \pm}, T, H\right) .
\end{aligned}
$$

The NLO mass correction $a_{0}$ reads

$$
a_{0}=\frac{\mathrm{d}\left(M_{\pi}^{0}\right)^{2}}{\mathrm{~d} M_{\pi}^{2}}=1+\frac{K_{1}}{F^{2}}+\frac{M_{\pi}^{2}}{F^{2}} \frac{\mathrm{d} K_{1}}{\mathrm{~d} M_{\pi}^{2}}
$$

where the integral $\mathrm{d} K_{1} / \mathrm{d} M_{\pi}^{2}$ is

$$
\frac{\mathrm{d} K_{1}}{\mathrm{~d} M_{\pi}^{2}}=-\frac{1}{16 \pi^{2}} \int_{0}^{\infty} \mathrm{d} \rho \exp \left(-\frac{M_{\pi}^{2}}{|q H|} \rho\right)\left(\frac{1}{\sinh (\rho)}-\frac{1}{\rho}\right) .
$$

In Fig. 6 we plot the scaled finite-temperature quark condensate, i.e., the dimensionless quantity

$$
-\left(q_{1}+q_{2} \frac{T^{2}}{F^{2}}\right)
$$

in terms of magnetic field strength $\left(m_{H}\right)$ and pion mass $(m)$ for the two temperatures $T=\{54,108\} \mathrm{MeV}$. The condensate grows as magnetic field strength or pion masses increase, but the dependence on pion mass is more pronounced.

\footnotetext{
${ }^{8}$ The quantity $\langle 0|\bar{q} q| 0\rangle_{0}$ is the quark condensate in the chiral limit at zero temperature and zero magnetic field.
}

To better appreciate the impact of the magnetic field-as for the entropy density - we subtract the $H=0$ portion and define the dimensionless quantity

$$
\langle\bar{q} q\rangle_{H}^{T}=\frac{c_{0}}{T^{2}}\left\{\langle\bar{q} q\rangle^{T}-\left.\langle\bar{q} q\rangle^{T}\right|_{H=0}\right\}
$$

where

$$
c_{0}=\frac{F^{2}}{\langle 0|\bar{q} q| 0\rangle_{0}}\left(1-\frac{M_{\pi}^{2}}{32 \pi^{2} F^{2}}\left(2 \bar{l}_{3}-1\right)\right)^{-1} .
$$

Notice that $\langle\bar{q} q\rangle_{H}^{T}$ captures the effect of the magnetic field in the finite-temperature quark condensate.

Inspecting the corresponding Fig. 7, it now becomes obvious that $\langle\bar{q} q\rangle_{H}^{T}$-i.e., the subtracted finite-temperature order parameter-is correlated with the subtracted entropy density (cf. Fig. 2): the enhancement of the order parameter caused by the magnetic field is reflected in the decrease of the entropy density. Note that in either subtracted quantity the influence of the magnetic field is largest in the chiral limit.

Let us finally discuss the connection between quark condensate and entropy density at the physical point $M_{\pi}=140 \mathrm{MeV}$. On the lhs of Fig. 8 we depict the scaled finite-temperature QCD quark condensate,

$$
-\left(q_{1}+q_{2} \frac{T^{2}}{F^{2}}\right)
$$



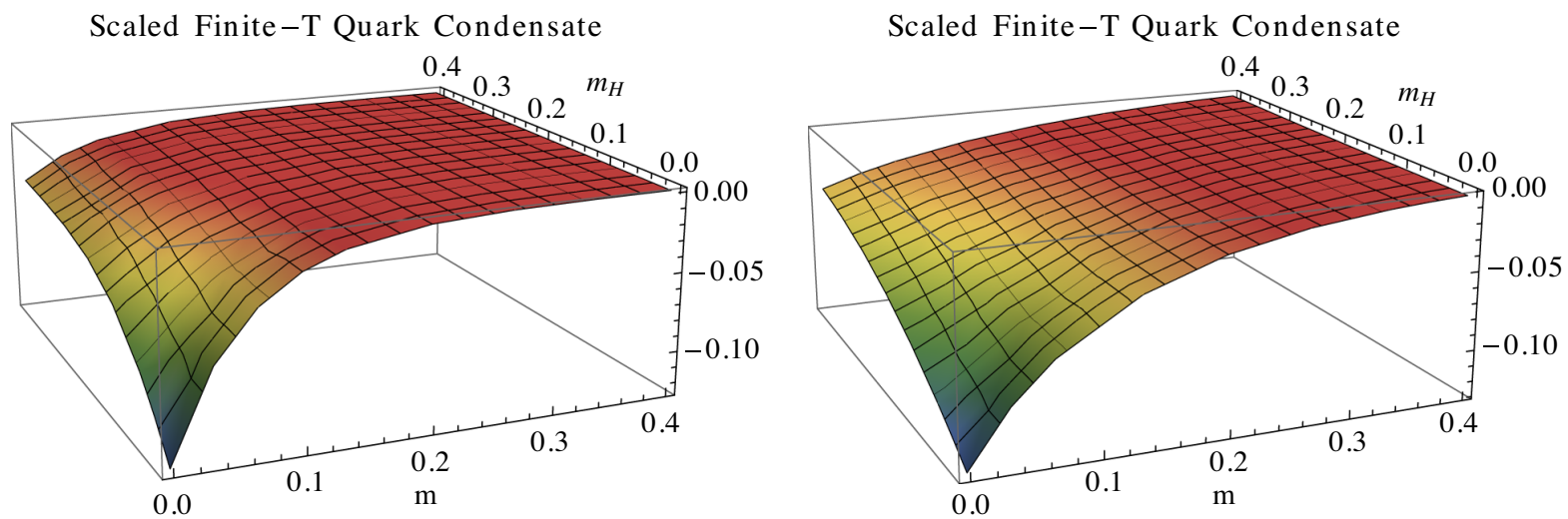

FIG. 6. Scaled finite-temperature quark condensate: Sum of one- and two-loop contributions for $T=54 \mathrm{MeV}$ (left) and $T=108 \mathrm{MeV}$ (right) in terms of the dimensionless parameters $m$ (pion mass) and $m_{H}$ (magnetic field strength).

in function of magnetic field strength and temperature. The influence of the magnetic field is barely visible in comparison to the predominant dependence on temperature. This motivates us to consider the subtracted QCD quark condensate,

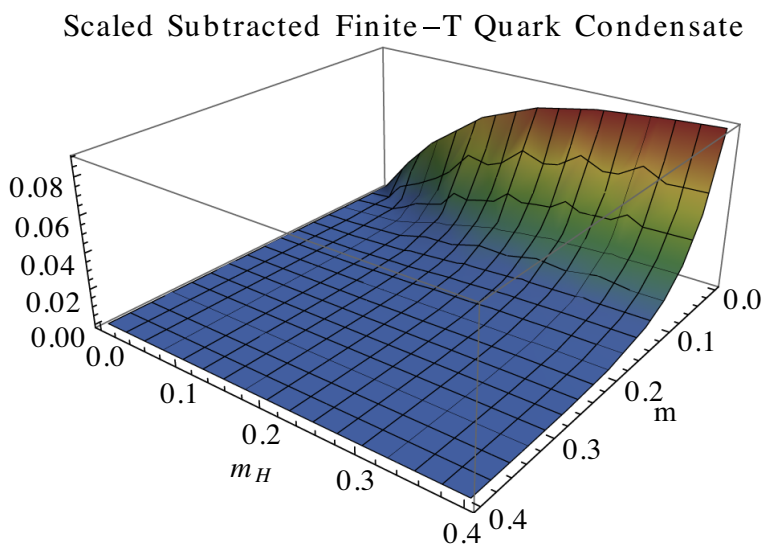

$$
\langle\bar{q} q\rangle_{H}^{T},
$$

that we show on the rhs of Fig. 8. Now the correlation between order parameter and entropy density (cf. rhs of Fig. 3) becomes obvious: the magnetic field enhances the

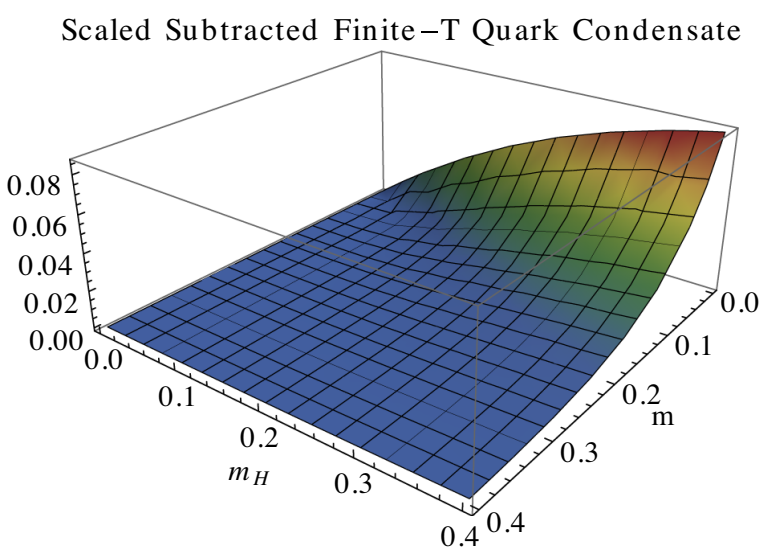

FIG. 7. Scaled subtracted finite-temperature quark condensate $\langle\bar{q} q\rangle_{H}^{T}$ : Sum of one- and two-loop contributions for $T=54 \mathrm{MeV}$ (left) and $T=108 \mathrm{MeV}$ (right) in terms of the dimensionless parameters $m$ (pion mass) and $m_{H}$ (magnetic field strength).

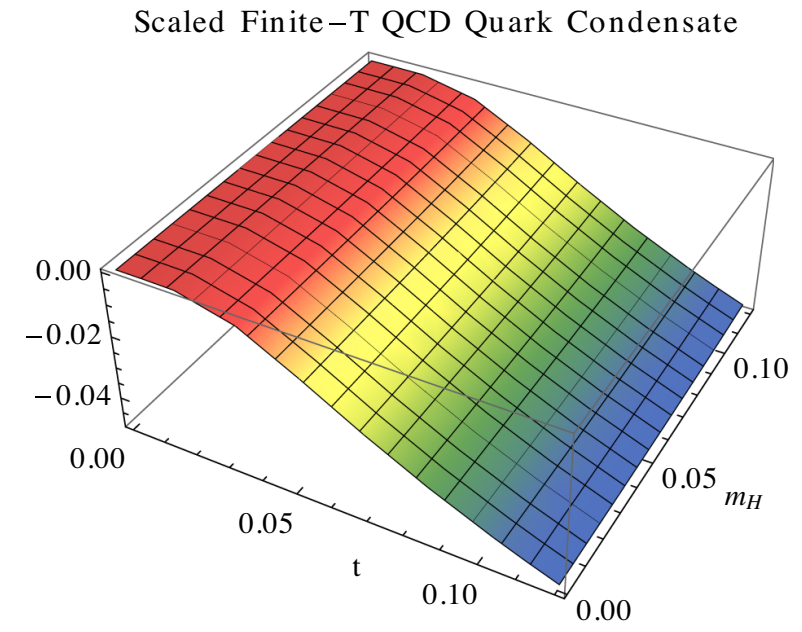

\section{Scaled Subtracted Finite-T QCD Quark Condensate}

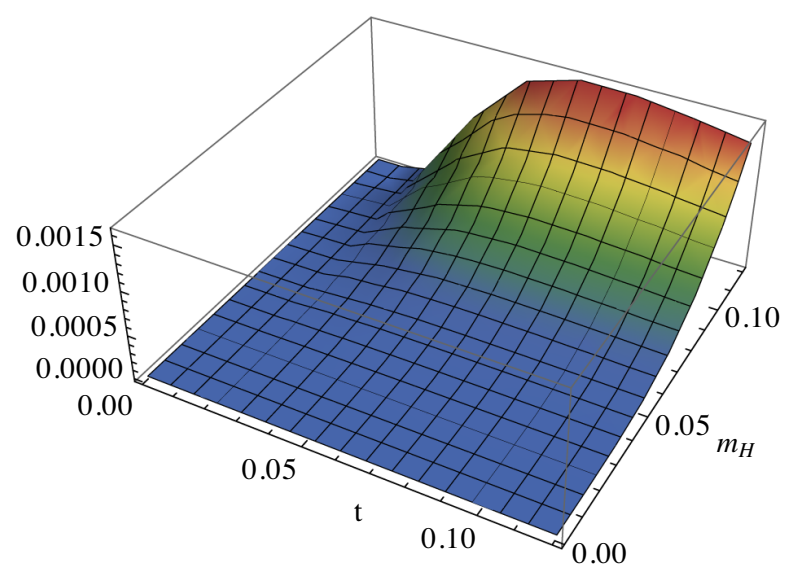

FIG. 8. Scaled finite-temperature QCD quark condensate in terms of the dimensionless parameters $m_{H}$ and $t$. Left: full contribution $-\left(q_{1}+q_{2} T^{2} / F^{2}\right)$. Right: subtracted contribution $\langle\bar{q} q\rangle_{H}^{T}$. 
finite-temperature quark condensate which is reflected in the decrease of the entropy density. It should be noted that the correlation is not one-to-one, but just is of qualitative nature. For example, as Fig. 8 illustrates, the effect of the magnetic field is most pronounced around $t \approx 0.085$, or $T \approx 90 \mathrm{MeV}$, while for the entropy density the most distinct drop occurs around $T \approx 40 \mathrm{MeV}$.

The question is whether this correlation-that is obvious in the plots-has any physical significance. To shed light on this issue let us consider a condensed matter system that behaves in an analogous way. More precisely, we refer to antiferromagnets that are subjected to magnetic and staggered fields. In antiferromagnets, instead of a spontaneously broken chiral symmetry, we have a spontaneously broken rotational symmetry-O(3) $\rightarrow O(2)$ - giving rise to two magnons (spin-wave branches) that are the respective Goldstone bosons. The order parameter is the staggered magnetization (the analog of the quark condensate) and the explicit symmetry breaking parameter is the staggered field (the analog of up- and down-quark mass). Within magnon effective field theory, i.e., the condensed matter analog of pion effective field theory, the entropy density shift as well as the finite-temperature staggered magnetization shift for this condensed matter system have been calculated very recently [50]. The crucial observation is that in antiferromagnets subjected to mutually orthogonal staggered and magnetic fields, which represents the most studied configuration, entropy shift and finite-temperature staggered magnetization shift are also correlated: as temperature grows, the entropy shift is negative while the order parameter shift is positive-much like in QCD. To reveal the phenomenon it was crucial to consider the respective shift in the observables caused by the magnetic field, i.e., to first subtract the $H=0$ portions in the entropy density and finite-temperature staggered magnetization-as we did so in the QCD entropy density and finite-temperature quark condensate, Eqs. (3.11) and Eqs. (5.7), respectively.

Now in the case of antiferromagnets, the meaning of the order parameter is intuitively clear and straightforward: the staggered magnetization measures the extent of spin antialignment. Naively, in the ground state (Néel state) of a twosublattice antiferromagnet, on one sublattice all spins point up and on the other sublattice all spins point down. This clearly represents a highly ordered spin configuration. The combined effect of magnetic field, temperature and staggered field (that is held constant, much like the quark masses are fixed at their physical values in the real world) perturb this antiparallel spin arrangement. So here it is intuitively clear that the correlation between entropy shift and staggered magnetization shift is not coincidental, but is of physical significance: both quantities-finite-temperature staggered magnetization and entropy density - measure the extent of order in the spin arrangement. Stronger antialignment of the spins-as measured by the finite-temperature staggered magnetization-corresponds to a spin configuration of higher order which is unambiguously reflected in the negative entropy density shift.

Based on this analogy with antiferromagnets, the claim is that the correlation in QCD that we observe between finitetemperature quark condensate and entropy density shift, also is of physical significance. In analogy to the staggered magnetization order parameter that measures the magnitude of (anti)alignment in spin space, here the quark condensate measures the "extent of order in flavor space." At finite temperature and in presence of a magnetic field, the quark condensate is enhanced as we observed on the rhs of Fig. 8. This enhancement of order is then indeed reflected in a negative entropy density shift according to the rhs of Fig. 3. Our speculation is that this correlation between entropy shift and quark condensate shift is not coincidental, but physically significant. The phenomenon may be studied with other nonperturbative methods such as QCD sum rules or lattice QCD.

\section{CONCLUSIONS}

Within the framework of two-flavor chiral perturbation theory in a magnetic background, we have derived the twoloop representations for the entropy density and the magnetization. In various figures we have explored how these quantities depend on temperature, magnetic field strength and pion mass.

We observe that the entropy density-at fixed temperature-drops when the magnetic field becomes stronger and that its decrease is most pronounced in the chiral limit. At the physical point $M_{\pi}=140 \mathrm{MeV}$, the decrease of the entropy density is most distinct around $T \approx 40 \mathrm{MeV}$. The lowering of the entropy density in an external magnetic field has also been reported in modelbased studies. Here, however, we have provided a fully systematic investigation.

The magnetization at finite temperature is negative in the entire parameter domain accessible by CHPT, i.e., $T, M_{\pi}, \sqrt{|q H|} \lesssim 0.2 \mathrm{GeV}$. Its dependence on magnetic field strength is nontrivial: the magnitude of the finite-temperature magnetization initially grows as the magnetic field increases, but then declines in stronger magnetic fields. This behavior is most distinct in the chiral limit. In the real world $\left(M_{\pi}=140 \mathrm{MeV}\right)$ the $\mathrm{QCD}$ vacuum behaves as a diamagnetic medium at low temperatures and weak magnetic fields, in accordance with lattice QCD and model-based studies.

Finally we have addressed the connection between the behavior of the entropy density and the finite-temperature quark condensate in a magnetic field. The connection becomes most obvious when the $H=0$ pieces in either quantity are subtracted, such that the effect of the magnetic field is revealed: we then observe that the enhancement of the finite-temperature quark condensate in a magnetic field is reflected in a decrease of the entropy density. Based on an analogy with antiferromagnets subjected to mutually orthogonal magnetic and staggered fields, we have 
speculated that the correlation between quark condensate shift and entropy density shift is not coincidental, but of physical significance.

Although the thermomagnetic properties of quantum chromodynamics in a homogeneous external magnetic field have been explored before by various authors, a comprehensive investigation of the entropy density and the magnetization in the regime of low temperatures and weak magnetic fields seems to be lacking. Here we have provided a fully systematic analysis relying on two-flavor chiral perturbation theory.

While we have focused on effects emerging at finite temperature, it would be interesting to also include zero-temperature effects into the discussion. This, however, requires a detailed analysis of the zero-temperature vacuum polarization. A detailed investigation including the magnetic susceptibility and the associated diamagnetic and paramagnetic phases-as well as an extensive comparison with the pertinent literature-is given in the companion paper [49]. It would also be interesting to extend the present case to three-flavor chiral perturbation theory, i.e., to include kaons and the $\eta$-particle. An even more ambitious task is to elevate the present two-loop analysis to the three-loop level. Corresponding work is in progress.

\section{ACKNOWLEDGMENTS}

The author thanks J. Bijnens and H. Leutwyler for correspondence.
[1] P. V. Buividovich, M. N. Chernodub, E. V. Luschevskaya, and M. I. Polikarpov, Nucl. Phys. B826, 313 (2010).

[2] G. S. Bali, F. Bruckmann, G. Endrödi, F. Gruber, and A. Schäfer, J. High Energy Phys. 04 (2013) 130.

[3] G. S. Bali, F. Bruckmann, G. Endrödi, S. D. Katz, and A. Schäfer, J. High Energy Phys. 08 (2014) 177.

[4] G. S. Bali, F. Bruckmann, G. Endrödi, and A. Schäfer, Phys. Rev. Lett. 112, 042301 (2014).

[5] V. V. Braguta, M. N. Chernodub, A. Y. Kotov, A. V. Molochkov, and A. A. Nikolaev, Phys. Rev. D 100, 114503 (2019).

[6] O. Scavenius, Á. Mócsy, I. N. Mishustin, and D. H. Rischke, Phys. Rev. C 64, 045202 (2001).

[7] S. Fayazbakhsh and N. Sadooghi, Phys. Rev. D 90, 105030 (2014).

[8] R. L. S. Farias, V. S. Timóteo, S. S. Avancini, M. B. Pinto, and G. Krein, Eur. Phys. J. A 53, 101 (2017).

[9] S. S. Avancini, R. L. S. Farias, M. B. Pinto, T. E. Restrepo, and W. R. Tavares, Phys. Rev. D 103, 056009 (2021).

[10] S. Chakrabarty, Phys. Rev. D 54, 1306 (1996).

[11] T. D. Cohen and E. S. Werbos, Phys. Rev. C 80, 015203 (2009).

[12] B.-J. Schäfer, M. Wagner, and J. Wambach, Phys. Rev. D 81, 074013 (2010).

[13] G. Endrödi, J. High Energy Phys. 04 (2013) 023.

[14] Y. Tsue, J. da Providencia, C. Providencia, M. Yamamura, and H. Bohr, Prog. Theor. Exp. Phys. (2015), 103 D01.

[15] V. Skalozub and P. Minaiev, arXiv:1612.00216.

[16] L.-G. Pang, G. Endrödi, and H. Petersen, Phys. Rev. C 93, 044919 (2016).

[17] A. N. Tawfik, A. M. Diab, N. Ezzelarab, and A. G. Shalaby, Adv. High Energy Phys. 2016, 1381479 (2016).

[18] S. Rath and B. K. Patra, J. High Energy Phys. 12 (2017) 098.

[19] A. N. Tawfik, A. M. Diab, and M. T. Hussein, J. Exp. Theor. Phys. 126, 620 (2018).
[20] B. Karmakar, R. Ghosh, A. Bandyopadhyay, N. Haque, and M. G. Mustafa, Phys. Rev. D 99, 094002 (2019).

[21] X. Li, W. Fu, and Y. Liu, Phys. Rev. D 99, 074029 (2019).

[22] S. Rath and B. K. Patra, Eur. Phys. J. A 55, 220 (2019).

[23] S. Koothottil and V. M. Bannur, Phys. Rev. C 102, 015206 (2020).

[24] C. P. Hofmann, Phys. Rev. D 99, 014030 (2019).

[25] C. P. Hofmann, Phys. Rev. D 101, 114031 (2020).

[26] C. P. Hofmann, Phys. Rev. D 102, 094010 (2020).

[27] C. Bonati, M. D'Elia, M. Mariti, F. Negro, and F. Sanfilippo, Phys. Rev. D 89, 054506 (2014).

[28] L. Levkova and C. DeTar, Phys. Rev. Lett. 112, 012002 (2014).

[29] G. S. Bali, G. Endrödi, and S. Piemonte, J. High Energy Phys. 07 (2020) 183.

[30] K. Kamikado and T. Kanazawa, J. High Energy Phys. 01 (2015) 129.

[31] H. Leutwyler, in Hadron Physics 94-Topics on the Structure and Interaction of Hadronic Systems, edited by V. E. Herscovitz, C. A. Z. Vasconcellos, and E. Ferreira (World Scientific, Singapore, 1995), p. 1.

[32] A. V. Manohar, in Perturbative and Nonperturbative Aspects of Quantum Field Theory, edited by H. Latal and W. Schweiger (Springer, New York, 1997), p. 311.

[33] S. Scherer, Adv. Nucl. Phys. 27, 277 (2003).

[34] J. L. Goity, Czech. J. Phys. 51, B35 (2001).

[35] T. Brauner, Symmetry 2, 609 (2010).

[36] I. A. Shushpanov and A. V. Smilga, Phys. Lett. B 402, 351 (1997).

[37] N. O. Agasian and I. A. Shushpanov, Phys. Lett. B 472, 143 (2000).

[38] N. O. Agasian, Phys. Lett. B 488, 39 (2000).

[39] N. O. Agasian and I. A. Shushpanov, J. High Energy Phys. 10 (2001) 006.

[40] T. D. Cohen, D. A. McGady, and E. S. Werbos, Phys. Rev. C 76, 055201 (2007).

[41] E. S. Werbos, Phys. Rev. C 77, 065202 (2008). 
[42] N. O. Agasian and S. M. Fedorov, Phys. Lett. B 663, 445 (2008).

[43] J. O. Andersen, Phys. Rev. D 86, 025020 (2012).

[44] J. O. Andersen, J. High Energy Phys. 10 (2012) 005.

[45] T. Brauner and S. V. Kadam, J. High Energy Phys. 11 (2017) 103.
[46] S. Aoki et al., Eur. Phys. J. C 80, 113 (2020).

[47] P. Gerber and H. Leutwyler, Nucl. Phys. B321, 387 (1989).

[48] C. P. Hofmann (to be published).

[49] C. P. Hofmann, Phys. Lett. B 818, 136384 (2021).

[50] C. P. Hofmann (to be published). 